\title{
Cumulative Error Estimation from Noisy Relative Measurements
}

\author{
Feihu Zhang, Carsten Simon, Guang Chen, Christian Buckl and Alois Knoll
}

\begin{abstract}
Odometry is important for autonomous vehicle in scenarios where GPS is either unavailable or only intermittently available. However, in a large scale environment, it often generalizes unbounded cumulative error when the vehicle unconsciously moves. This paper analyzes how the cumulative error grows according to the noisy relative measurements. An unbounded drift model is proposed to represent the cumulative error, where its probability distribution is described by the corresponding expectation and variance. Compared to other approaches, it presents a recursive cumulative error expression in absence of the true positions, which has great potentials in various domains, e. g. path planning, odmetry based localization.
\end{abstract}

Both experiments and cases are conducted to not only verify the accuracy of the proposed model, but also illustrate the potentials in related domains.

\section{INTRODUCTION}

Within the past decade, accurate global localization has become a hot issue in the intelligent vehicle research domain, not only for developing advanced driver assistance system, but also for autonomous driving.

Since GPS is susceptible to interference or even not fully available (tunnels, indoor environments, mountainous forested environments), odometry based localization is taken as a supplement solution to calculate the position of the vehicle by using e.g. vision based sensors (cameras, LIDARs, etc) [1], [2], [3], wheel sensors [4] and inertial sensors (gyroscopes and accelerometers) [5], [6], [7].

Although odometry provides high accuracy localizations, drift accumulation (cumulative error) is still an issue which is caused by noisy relative measurements. A number of papers have claimed, without proof, the cumulative error grows nonlinearly with distance or time [8], [9]. However, a rigorous analysis of the growth rate is still missing.

Methods which eliminate the drift have been demonstrated by using terrain maps [10] and visual landmark recognition [11], where the calibration is executed after following a suitable trajectory. However, in large scale urban environments, the computational complexity is a challenge. Vorst et. al. [12] present a method which uses RFID technique to improve the precision. In their research, the localization module is initialized periodically by the RFID, with the goal of keeping the drift bounded. The proposed approach exhibits good robustness under various scenarios. However, it gives no insight about how to optimally distribute the RFID, which relates to the growth of the cumulative error.

Feihu Zhang, Guang Chen and Alois Knoll are with the Technische Universität München, Garching bei München, Germany, e-mail: feihu.zhang@tum.de, \{guang, knoll $\} @$ in.tum.de.

Christian Buckl and Carsten Simon are with fortiss $\mathrm{GmbH}$, München, e-mail: \{buckl,simon\}@fortiss.org.
In this paper, we examine the growth rate of the cumulative error by noisy relative measurements. In addition, the proposed formulas are also verified numerically through Monte-Carlo simulation. The contribution is that it provides a feasible solution to evaluate the cumulative error recursively without utilizing the true positions, which is either practical or theoretical significance. Furthermore, we also exhibit the corresponding potentials in the case of path planning, odmetry based localization.

An off-the-shelf platform is used to provide data from real traffic scenarios [13]. The platform is equipped with sensors to record the relative measurements (velocity and orientation) at each step. The experimental results indicate that the proposed method yields precise estimations.

The remainder of this paper is structured as follows: Sec. II briefly describes the related work. Sec. III introduces more details about the proposed drift models. Sec. IV presents experimental results with Monte-Carlo simulation while Sec. $\mathrm{V}$ exploits the error estimations in the related domains. Finally, the paper is concluded in Sec.VI.

\section{RELATED WORK}

The work by Smith and Cheesman [14], Su et. al. [15] and Wang et. al. [16] introduce recursive expressions for the covariance of the pose estimation error. They utilize the first order of the $\mathrm{BCH}$ (Baker-Cambell-Hausdorff) formula by assuming that the errors are small. Zhou et. al. [17] describe the corresponding error by utilizing its probability density function. Ruyi et. al. [18] utilize an unbounded model to represent the cumulative error. However, they don't analyze asymptotic behavior of the error.

Olson et. al. [19] illustrate that the cumulative error grows super-linearly. They also exhibit that the cumulative error grows as $O\left(d^{3 / 2}\right)$, where $d$ is the distance. However, there is no specific models and parameters. A parametric statistical model of the cumulative error is proposed in [20], which provides an exact formula for both expectation and variance of the cumulative error. It calculates the exact formula from two special trajectories-straight line and periodic curve, which is still an issue on representing the real environment.

Calibration based methods are also investigated to eliminate the error, which calculates the differences between the actual point and the estimated point. Borenstein et. al. analyze the possible source of odometry error [21]. Kelly proposes a general solution using linearized error equation for any trajectory and model [22]. Antonelli et. al. present a calibration method based on the least-squares technique [23]. These methods can be classified into off-line methods since they are executed after following a suitable test trajectory. On 


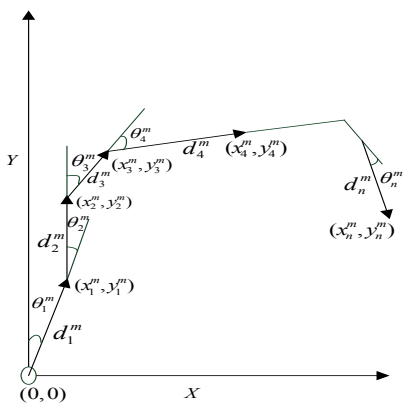

Fig. 1. Relationship between the relative measurements and the positions

the other hand, calibration is executed through continuous steps when a robot is able to estimate the pose. Roy et. al. suggest an algorithm to recalibrate the robot according to the probabilistic distribution [24]. This method estimates both the robot configuration and the parameters simultaneously. In despite of the merits, there is a significant problem. The performance depends on the prior information, which is often unavailable in an unknown environment.

\section{iII. Cumulative Error Estimation}

In this section, we analyze how the cumulative error grows according to the noisy relative measurements. An unbounded drift model is also proposed, where its probability distribution is described by the corresponding expectation and variance.

\section{A. Problem statement}

The vehicle's position is estimated by accumulating the relative measurements during the whole process. Fig. 1 illustrates the relationship between the noisy relative measurements $\left(\theta_{n}^{m}, d_{n}^{m}\right)$ and the corresponding position $\left(x_{n}^{m}, y_{n}^{m}\right)$, where $n$ is the time index, $m$ is short acronym for the measurement. $d$ and $\theta$ represents the corresponding velocity and orientation between consecutive frames. Measurements $\bar{\theta}_{n}$ and $\bar{d}_{n}$ are defined with respect to the true values as

$$
\theta_{n}^{m}=\bar{\theta}_{n}+\tilde{\theta}_{n} ; d_{n}^{m}=\bar{d}_{n}+\tilde{d}_{n}
$$

where $\tilde{\theta}_{n}$ and $\tilde{d}_{n}$ are assumed to be independent with zero mean and standard deviations $\delta_{\theta}, \delta_{d}$. The trajectory is calculated by the following equations in practice:

$$
x_{n}^{m}=\sum_{i=1}^{n}\left(d_{i}^{m} \sin \sum_{j=1}^{i} \theta_{j}^{m}\right) ; y_{n}^{m}=\sum_{i=1}^{n}\left(d_{i}^{m} \cos \sum_{j=1}^{i} \theta_{j}^{m}\right)
$$

Fig. 2 exhibits the trajectories based on the odometry, where the real data is calculated with the true values:

$$
\bar{x}_{n}=\sum_{i=1}^{n}\left(\bar{d}_{i} \sin \sum_{j=1}^{i} \bar{\theta}_{j}\right) ; \bar{y}_{n}=\sum_{i=1}^{n}\left(\bar{d}_{i} \cos \sum_{j=1}^{i} \bar{\theta}_{j}\right)
$$

As illustrated in Fig. 2, the cumulative error grows unbounded with time increasing. It is a challenge to estimate the cumulative error only using the noisy measurements. However, the corresponding statistical properties are feasible.

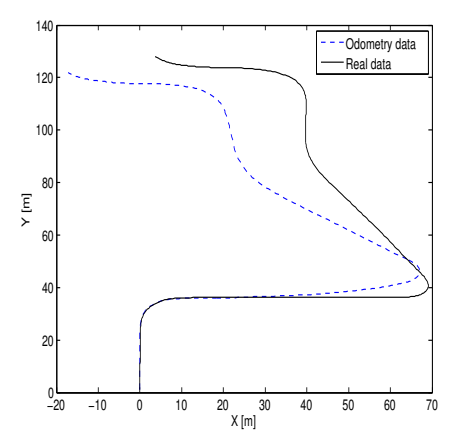

Fig. 2. Odometry result and the real data

In this paper, we estimate both expectation and variance of the cumulative error by utilizing the statistical properties of the noises.

\section{B. True Error Statistics}

The odometry result can also be represented as a combination of the true value and the cumulative error :

$$
\begin{aligned}
& x_{n}^{m}=\bar{x}_{n}+\tilde{x}_{n}=\sum_{i=1}^{n}\left[\left(\bar{d}_{i}+\tilde{d}_{i}\right) \sin \left(\sum_{j=1}^{i}\left(\bar{\theta}_{j}+\tilde{\theta}_{j}\right)\right)\right] \\
& =\left[\sum_{i=1}^{n} \bar{d}_{i}+\sum_{i=1}^{n} \tilde{d}_{i}\right] \cdot\left[\sin \sum_{j=1}^{i} \bar{\theta}_{j} \cos \sum_{j=1}^{i} \tilde{\theta}_{j}+\cos \sum_{j=1}^{i} \bar{\theta}_{j}\right. \\
& \left.\cdot \sin \sum_{j=1}^{i} \tilde{\theta}_{j}\right] \\
& y_{n}^{m}=\bar{y}_{n}+\tilde{y}_{n}=\sum_{i=1}^{n}\left[\left(\bar{d}_{i}+\tilde{d}_{i}\right) \cos \left(\sum_{j=1}^{i}\left(\bar{\theta}_{j}+\tilde{\theta}_{j}\right)\right)\right] \\
& =\left[\sum_{i=1}^{n} \bar{d}_{i}+\sum_{i=1}^{n} \tilde{d}_{i}\right] \cdot\left[\cos \sum_{j=1}^{i} \bar{\theta}_{j} \cos \sum_{j=1}^{i} \tilde{\theta}_{j}-\sin \sum_{j=1}^{i} \bar{\theta}_{j}\right. \\
& \left.\cdot \sin \sum_{j=1}^{i} \tilde{\theta}_{j}\right]
\end{aligned}
$$

Rearranging the above equations we can obtain the mathematical expression of the cumulative error:

$$
\begin{aligned}
& \tilde{x}_{n}=\sum_{i=1}^{n} \bar{d}_{i}\left[\sin \sum_{j=1}^{i} \bar{\theta}_{j}\left(\cos \sum_{j=1}^{i} \tilde{\theta}_{j}-1\right)+\cos \sum_{j=1}^{i} \bar{\theta}_{j} \sin \sum_{j=1}^{i} \tilde{\theta}_{j}\right] \\
& +\sum_{i=1}^{n} \tilde{d}_{i}\left[\sin \sum_{j=1}^{i} \bar{\theta}_{j} \cos \sum_{j=1}^{i} \tilde{\theta}_{j}+\cos \sum_{j=1}^{i} \bar{\theta}_{j} \sin \sum_{j=1}^{i} \tilde{\theta}_{j}\right] \\
& \tilde{y}_{n}=\sum_{i=1}^{n} \bar{d}_{i}\left[\cos \sum_{j=1}^{i} \bar{\theta}_{j}\left(\cos \sum_{j=1}^{i} \tilde{\theta}_{j}-1\right)-\sin \sum_{j=1}^{i} \bar{\theta}_{j} \sin \sum_{j=1}^{i} \tilde{\theta}_{j}\right] \\
& +\sum_{i=1}^{n} \tilde{d}_{i}\left[\cos \sum_{j=1}^{i} \bar{\theta}_{j} \cos \sum_{j=1}^{i} \tilde{\theta}_{j}-\sin \sum_{j=1}^{i} \bar{\theta}_{j} \sin \sum_{j=1}^{i} \tilde{\theta}_{j}\right]
\end{aligned}
$$

The cumulative error is dependent and each depends on the true values. The expectation and variance is estimated according to the statistical properties of the relative measurements. 
TABLE I

REPRESENTS OF THE PARAMETERS

\begin{tabular}{|c|c|}
\hline $\mathbf{A}$ & $\sum_{i=1}^{n} \bar{d}_{i}^{2}\left[\sin ^{2} \sum_{j=1}^{i} \bar{\theta}_{j}\left(0.5 e^{-2 i \delta_{\theta}^{2}}+1.5-2 e^{-\frac{i \delta_{\theta}^{2}}{2}}\right)+0.5 \cos ^{2} \sum_{j=1}^{i} \bar{\theta}_{j}\left(e^{-2 i \delta_{\theta}^{2}}+1\right)\right]$ \\
\hline B & $\begin{array}{l}2 \sum_{i=1}^{n-1} \sum_{p=1+i}^{n} \bar{d}_{i} \bar{d}_{p}\left\{\sin ^{2} \sum_{j=1}^{i} \bar{\theta}_{j} \cos \Delta \bar{\theta}\left[-e^{-0.5 i \delta_{\theta}^{2}}+0.5\left(1+e^{-2 i \delta_{\theta}^{2}}\right) e^{-0.5 \delta_{\theta}^{2}(p-i)}+1-e^{-0.5 i \delta_{\theta}^{2}} e^{-0.5 \delta_{\theta}^{2}(p-i)}\right]\right. \\
+\sin \sum_{j=1}^{i} \bar{\theta}_{j} \sin \Delta \bar{\theta} \cos \sum_{j=1}^{i} \bar{\theta}_{j}\left[-e^{-0.5 i \delta_{\theta}^{2}}+0.5\left(1+e^{-2 i \delta_{\theta}^{2}}\right) e^{-0.5 \delta_{\theta}^{2}(p-i)}+1-e^{-0.5 i \delta_{\theta}^{2}} e^{-0.5 \delta_{\theta}^{2}(p-i)}\right. \\
\left.\left.-0.5\left(1-e^{-2 i \delta_{\theta}^{2}}\right) e^{-0.5 \delta_{\theta}^{2}(p-i)}\right]+\cos ^{2} \sum_{j=1}^{i} \bar{\theta}_{j} \cos \Delta \bar{\theta} 0.5\left(1-e^{-2 i \delta_{\theta}^{2}}\right) e^{-0.5 \delta_{\theta}^{2}(p-i)}\right\}\end{array}$ \\
\hline C & $\sum_{i=1}^{n}\left[0.5 \sin ^{2} \sum_{j=1}^{i} \bar{\theta}_{j}\left(1+e^{-2 i \delta_{\theta}^{2}}\right)+0.5 \cos ^{2} \sum_{j=1}^{i} \bar{\theta}_{j}\left(1-e^{-2 i \delta_{\theta}^{2}}\right)\right]$ \\
\hline D & $\sum_{i=1}^{n} \bar{d}_{i}^{2}\left[\cos ^{2} \sum_{j=1}^{i} \bar{\theta}_{j}\left(0.5 e^{-2 i \delta_{\theta}^{2}}+1.5-2 e^{-\frac{i \delta_{\theta}^{2}}{2}}\right)+0.5 \sin ^{2} \sum_{j=1}^{i} \bar{\theta}_{j}\left(-e^{-2 i \delta_{\theta}^{2}}+1\right)\right]$ \\
\hline $\mathbf{E}$ & $\begin{array}{l}2 \sum_{i=1}^{n-1} \sum_{p=1+i}^{n} \bar{d}_{i} \bar{d}_{p}\left\{\cos ^{2} \sum_{j=1}^{i} \bar{\theta}_{j} \cos \Delta \bar{\theta}\left[-e^{-0.5 i \delta_{\theta}^{2}}+0.5\left(1+e^{-2 i \delta_{\theta}^{2}}\right) e^{-0.5 \delta_{\theta}^{2}(p-i)}+1-e^{-0.5 i \delta_{\theta}^{2}} e^{-0.5 \delta_{\theta}^{2}(p-i)}\right]\right. \\
+\sin \sum_{j=1}^{i} \bar{\theta}_{j} \sin \Delta \bar{\theta} \cos \sum_{j=1}^{i} \bar{\theta}_{j}\left[e^{-0.5 i \delta_{\theta}^{2}}+0.5\left(1-e^{-2 i \delta_{\theta}^{2}}\right) e^{-0.5 \delta_{\theta}^{2}(p-i)}-1+e^{-0.5 i \delta_{\theta}^{2}} e^{-0.5 \delta_{\theta}^{2}(p-i)}\right. \\
\left.\left.-0.5\left(1+e^{-2 i \delta_{\theta}^{2}}\right) e^{-0.5 \delta_{\theta}^{2}(p-i)}\right]+\sin ^{2} \sum_{j=1}^{i} \bar{\theta}_{j} \cos \Delta \bar{\theta} 0.5\left(1-e^{-2 i \delta_{\theta}^{2}}\right) e^{-0.5 \delta_{\theta}^{2}(p-i)}\right\}\end{array}$ \\
\hline $\mathbf{F}$ & $\sum_{i=1}^{n}\left[0.5 \cos ^{2} \sum_{j=1}^{i} \bar{\theta}_{j}\left(1+e^{-2 i \delta_{\theta}^{2}}\right)+0.5 \sin ^{2} \sum_{j=1}^{i} \bar{\theta}_{j}\left(1-e^{-2 i \delta_{\theta}^{2}}\right)\right]$ \\
\hline
\end{tabular}

Proposition 1: The expectation of $\tilde{x}_{n}$ and $\tilde{y}_{n}$ is calculated as follows:

$$
\begin{gathered}
\mu_{n}(\bar{\theta}, \bar{d})=\left[\begin{array}{c}
E\left[\tilde{x}_{n} \mid \bar{\theta}, \bar{d}\right] \\
E\left[\tilde{y}_{n} \mid \bar{\theta}, \bar{d}\right]
\end{array}\right] \\
=\left[\begin{array}{c}
\sum_{i=1}^{n} \bar{d}_{i}\left[\sin \sum_{j=1}^{i} \bar{\theta}_{j}\left(e^{-\frac{i \delta_{\theta}^{2}}{2}}-1\right)\right] \\
\sum_{i=1}^{n} \bar{d}_{i}\left[\cos \sum_{j=1}^{i} \bar{\theta}_{j}\left(e^{-\frac{i \delta_{\theta}^{2}}{2}}-1\right)\right]
\end{array}\right]
\end{gathered}
$$

while the variance is

$$
\begin{aligned}
& \operatorname{var}(\tilde{x} \mid \bar{\theta}, \bar{d})=E\left[\tilde{x}^{2} \mid \bar{\theta}, \bar{d}\right]-E^{2}[\tilde{x} \mid \bar{\theta}, \bar{d}] \\
& =\mathbf{A}+\mathbf{B}+\mathbf{C}-E^{2}[\tilde{x} \mid \bar{\theta}, \bar{d}] \\
& \operatorname{var}(\tilde{y} \mid \bar{\theta}, \bar{d})=E\left[\tilde{y}^{2} \mid \bar{\theta}, \bar{d}\right]-E^{2}[\tilde{y} \mid \bar{\theta}, \bar{d}] \\
& =\mathbf{D}+\mathbf{E}+\mathbf{F}-E^{2}[\tilde{y} \mid \bar{\theta}, \bar{d}]
\end{aligned}
$$

Table I represents the mathematic expressions of the variances. Here $\Delta \theta$ represents the cumulated orientation $\sum_{k=i+1}^{p} \theta_{k}$.

\section{True Error Statistics in Practice}

Equations (4) and (5) are explicit expressions for the cumulative error. They have significant bias depending on the true value of the relative measurements, which is unavailable in practice. To make the results useful, the expected values of the true moments are evaluated conditioned on the noisy measurements. In this paper, the interest is to calculate the expected expectations and variances:

$$
\begin{aligned}
& E\left[\mu_{n} \mid \theta^{m}, d^{m}\right]=\mu_{m} \\
& E\left[\operatorname{var}\left(\tilde{x}_{n}\right) \mid \theta^{m}, d^{m}\right]=\operatorname{var}\left(\tilde{x}_{n}^{m}\right) \\
& E\left[\operatorname{var}\left(\tilde{y}_{n}\right) \mid \theta^{m}, d^{m}\right]=\operatorname{var}\left(\tilde{y}_{n}^{m}\right)
\end{aligned}
$$

Equation (6) is called the average true expectation and the average true variance.

The proof of proposition 2 is also provided in the Appendix. 


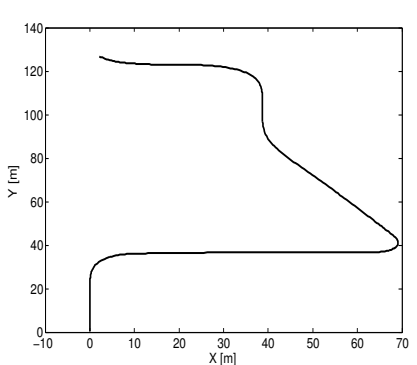

(a)

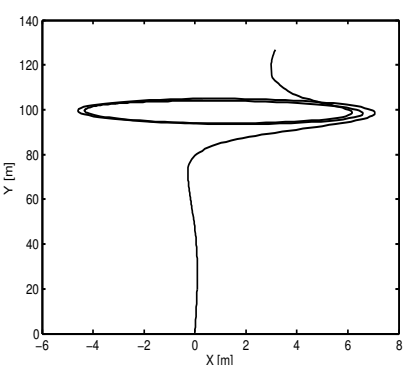

(b)
Fig. 3. The trajectories of the vehicle

Proposition 2: The error's true expectation and variance can be estimated as

$$
\begin{aligned}
& E\left[\tilde{x}_{n}^{m}\right]=\sum_{i=1}^{n} d_{i}^{m}\left(e^{-i \delta_{\theta}^{2}}-e^{\frac{-i \delta_{\theta}^{2}}{2}}\right) \sin \sum_{j=1}^{i} \theta_{j}^{m} \\
& E\left[\tilde{y}_{n}^{m}\right]=\sum_{i=1}^{n} d_{i}^{m}\left(e^{-i \delta_{\theta}^{2}}-e^{\frac{-i \delta_{\theta}^{2}}{2}}\right) \cos \sum_{j=1}^{i} \theta_{j}^{m} \\
& \operatorname{var}\left(\tilde{x}_{n}^{m}\right)=\mathbf{A}_{1}+\mathbf{B}_{1}+\mathbf{C}_{1}-E^{2}\left[\tilde{x}_{n}^{m}\right] \\
& \operatorname{var}\left(\tilde{y}_{n}^{m}\right)=\mathbf{D}_{1}+\mathbf{E}_{1}+\mathbf{F}_{1}-E^{2}\left[\tilde{y}_{n}^{m}\right]
\end{aligned}
$$

Table II also shows the mathematic expressions of the variances. Therefore, the statistical properties of the cumulative error are estimated, which has great potentials in practice.

\section{EXPERIMENTS}

In this section we estimate both expectation and variance of the cumulative error by conducting Monte-Carlo simulation. The relative measurements are acquired from the public dataset [13]. In our experiment, those noisy measurements are acquired by manually adding Gaussian white noises on the original data at each step. The distribution of the velocity noise is with the deviation of $0.1 \mathrm{~m}$ while the orientation noise is considered as an angular deviation of $0.1 \mathrm{rad}$.

Fig. 3 shows two trajectories including in the experiment which are measured by the GPS/IMU system.

Fig. 4 illustrates the proposed formulas compared with 1000 times Monte-Carlo simulation. The first row represents the trajectory in Fig. 3(a) while the second row represents Fig. 3(b). The first two columns illustrate the expectation of the cumulative error, where the rests illustrate the corresponding variance. As we can see from Fig. 4(a), 4(e), the expectation of the cumulative error in $X$ direction is almost zero at beginning. This is caused by the corresponding trajectory. Fig. 3(b) shows that the related trajectory is only extended in $Y$ direction during the same period. The influence of the noisy measurements in $X$ direction is therefore eliminated. The corresponding cumulative error grows dependent with the vehicle moves, which is also fitted to the physical models. Since the uncertainties grow explosively, the corresponding variances in Fig. 4 are quite huge compared with the first order moments. However, the proposed model still estimates well compared with the Monte-Carlo result.

Fig. 4 shows that the proposed model works well with the noisy measurements, which exhibits the high performance in the absence of true values.

\section{EXPLOITATION OF ERROR ESTIMATION}

The proposed model can also be considered as a solution to the path planning. Fig. 5(a) includes two trajectories on the ground plane with same lengths. The vehicle starts from the init point to the end point, executes the instructions at each step (velocity and orientation, which is pre-calculated by the planner). However, assuming the movements are distributed with Gaussian white noises, the position error is therefore existed (similar to the odometry, the position is localized by cumulating the movements during the process). The key issue is to find the minimum expectation of the position error. Fig. 5(b) and Fig. 5(c) illustrates the expectation of the error in $X$ and $Y$ direction estimated by the proposed model. Fig. 5(d) represents the position error by calculating the root square values from both directions. It is obvious that trajectory 2 has the minimum expectation of the position error compared with trajectory 1 , which helps the path planner by introducing a new cost mechanism.

The proposed model also has great potentials in other applications. For instance, Combining with the RFID technique to optimally distribute the RFID sensors in a large scale environment. As mentioned in Sec. I, Vorst presented a method by using RFID technique to improve the localization precision. However, there was no insight about how to optimally distribute the sensors, which related to the growth rate of the cumulative errors. Since the proposed model represents well with the cumulative error's statistical property, it becomes a feasible solution to optimally distribute the references.

In addition, it is a challenge to evaluate various odometry methods. Different trajectories cause the results deviating from the real status. However, the proposed model provides a natural bridge to evaluate the corresponding performances. Assuming each method's estimation uncertainty is known, the proposed formulas can evaluate the corresponding performance in various scenarios.

\section{CONCLUSION}

Modeling and analyzing the cumulative error from noisy relative measurements is either practical or theoretical significance. In this paper, we introduce a precise mathematical model to represent the statistical properties of the error. The model not only illustrates the corresponding error depending on the trajectory, but also calculates its expectation and variance. In comparison to the related work, the proposed approach recursively estimates the cumulative error in absence of the true values. Exploited work is also demonstrated to illustrate the huge potentials in the related domains.

The proposed method has been evaluated by Monte-Carlo simulation which performs well.

Future work focuses on the real implementation of the proposed approach. 
TABLE II

REPRESENTS OF THE PARAMETERS

\begin{tabular}{|c|c|}
\hline $\mathbf{A}_{1}$ & $\begin{array}{l}\sum_{i=1}^{n}\left(d_{i}^{m}\right)^{2}\left\{\left[0.5\left(1+e^{-2 i \delta \theta^{2}}\right) \sin ^{2} \sum_{j=1}^{i} \theta_{j}^{m}+0.5\left(1-e^{-2 i \delta \theta^{2}}\right) \cos ^{2} \sum_{j=1}^{i} \theta_{j}^{m}\right]\left(0.5 e^{-2 i \delta_{\theta}^{2}}+1.5-2 e^{-0.5 i \delta_{\theta}^{2}}\right)\right. \\
\left.+0.5\left(1+e^{-2 i \delta \theta^{2}}\right)\left[0.5\left(1+e^{-2 i \delta \theta^{2}}\right) \cos ^{2} \sum_{j=1}^{i} \theta_{j}^{m}+0.5\left(1-e^{-2 i \delta \theta^{2}}\right) \sin ^{2} \sum_{j=1}^{i} \theta_{j}^{m}\right]\right\}\end{array}$ \\
\hline $\mathbf{B}_{1}$ & $\begin{array}{l}2 \sum_{i=1}^{n-1} \sum_{p=1+i}^{n} d_{i}^{m} d_{p}^{m}\left\{\left[0.5\left(1+e^{-2 i \delta \theta^{2}}\right) \sin ^{2} \sum_{j=1}^{i} \theta_{j}^{m}+0.5\left(1-e^{-2 i \delta \theta^{2}}\right) \cos ^{2} \sum_{j=1}^{i} \theta_{j}^{m}\right]\left[\cos \Delta \theta^{m} e^{-0.5(p-i) \delta_{\theta}^{2}}\right][\ldots]\right. \\
+\left[\sin \sum_{j=1}^{i} \theta_{j}^{m} \sin \Delta \theta^{m} \cos \sum_{j=1}^{i} \theta_{j}^{m} e^{-2 i \delta \theta^{2}} e^{-0.5(p-i) \delta_{\theta}^{2}}\right][\ldots]+\left[0.5\left(1+e^{-2 i \delta \theta^{2}}\right) \cos ^{2} \sum_{j=1}^{i} \theta_{j}^{m}\right. \\
\left.\left.+0.5\left(1-e^{-2 i \delta \theta^{2}}\right) \sin ^{2} \sum_{j=1}^{i} \theta_{j}^{m}\right]\left[\cos \Delta \theta^{m} e^{-0.5(p-i) \delta_{\theta}^{2}}\right][\ldots]\right\}\end{array}$ \\
\hline $\mathbf{C}_{1}$ & $\begin{array}{l}\sum_{i=1}^{n}\left\{0.25\left(1+e^{-2 i \delta_{\theta}^{2}}\right)\left[\left(1+e^{-2 i \delta \theta^{2}}\right) \sin ^{2} \sum_{j=1}^{i} \theta_{j}^{m}+\left(1-e^{-2 i \delta \theta^{2}}\right) \cos ^{2} \sum_{j=1}^{i} \theta_{j}^{m}\right]+0.25\left(1-e^{-2 i \delta_{\theta}^{2}}\right)[(1+\right. \\
\left.\left.\left.e^{-2 i \delta \theta^{2}}\right) \cos ^{2} \sum_{j=1}^{i} \theta_{j}^{m}+\left(1-e^{-2 i \delta \theta^{2}}\right) \sin ^{2} \sum_{j=1}^{i} \theta_{j}^{m}\right]\right\}\end{array}$ \\
\hline $\mathbf{D}_{1}$ & $\begin{array}{l}\sum_{i=1}^{n}\left(d_{i}^{m}\right)^{2}\left\{\left[0.5\left(1+e^{-2 i \delta \theta^{2}}\right) \sin ^{2} \sum_{j=1}^{i} \theta_{j}^{m}+0.5\left(1-e^{-2 i \delta \theta^{2}}\right) \cos ^{2} \sum_{j=1}^{i} \theta_{j}^{m}\right]\left(1-e^{-2 i \delta \theta^{2}}\right)\right. \\
\left.+0.5\left(0.5 e^{-2 i \delta_{\theta}^{2}}+1.5-2 e^{-0.5 i \delta_{\theta}^{2}}\right)\left[0.5\left(1+e^{-2 i \delta \theta^{2}}\right) \cos ^{2} \sum_{j=1}^{i} \theta_{j}^{m}+0.5\left(1-e^{-2 i \delta \theta^{2}}\right) \sin ^{2} \sum_{j=1}^{i} \theta_{j}^{m}\right]\right\}\end{array}$ \\
\hline $\mathbf{E}_{1}$ & $\begin{array}{l}2 \sum_{i=1}^{n-1} \sum_{p=1+i}^{n} d_{i}^{m} d_{p}^{m}\left\{\left[0.5\left(1+e^{-2 i \delta \theta^{2}}\right) \cos ^{2} \sum_{j=1}^{i} \theta_{j}^{m}+0.5\left(1-e^{-2 i \delta \theta^{2}}\right) \sin ^{2} \sum_{j=1}^{i} \theta_{j}^{m}\right]\left[\cos \Delta \theta^{m} e^{-0.5(p-i) \delta_{\theta}^{2}}\right][\ldots]\right. \\
+\left[\sin \sum_{j=1}^{i} \theta_{j}^{m} \sin \Delta \theta^{m} \cos \sum_{j=1}^{i} \theta_{j}^{m} e^{-2 i \delta \theta^{2}} e^{-0.5(p-i) \delta_{\theta}^{2}}\right][\ldots]+\left[0.5\left(1+e^{-2 i \delta \theta^{2}}\right) \sin ^{2} \sum_{j=1}^{i} \theta_{j}^{m}+0.5\left(1-e^{-2 i \delta \theta^{2}}\right)\right. \\
\left.\left.\cdot \cos ^{2} \sum_{j=1}^{i} \theta_{j}^{m}\right]\left[\cos \Delta \theta^{m} e^{-0.5(p-i) \delta_{\theta}^{2}}\right][\ldots]\right\}\end{array}$ \\
\hline $\mathbf{F}_{1}$ & $\begin{array}{l}\sum_{i=1}^{n}\left\{0.25\left(1+e^{-2 i \delta_{\theta}^{2}}\right)\left[\left(1+e^{-2 i \delta \theta^{2}}\right) \cos ^{2} \sum_{j=1}^{i} \theta_{j}^{m}+\left(1-e^{-2 i \delta \theta^{2}}\right) \sin ^{2} \sum_{j=1}^{i} \theta_{j}^{m}\right]+0.25\left(1-e^{-2 i \delta_{\theta}^{2}}\right)[(1+\right. \\
\left.\left.\left.e^{-2 i \delta \theta^{2}}\right) \sin ^{2} \sum_{j=1}^{i} \theta_{j}^{m}+\left(1-e^{-2 i \delta \theta^{2}}\right) \cos ^{2} \sum_{j=1}^{i} \theta_{j}^{m}\right]\right\}\end{array}$ \\
\hline
\end{tabular}




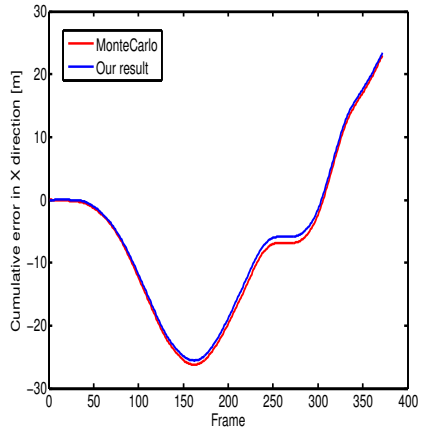

(a)

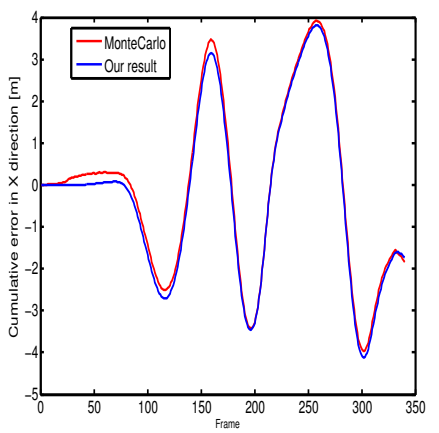

(e)

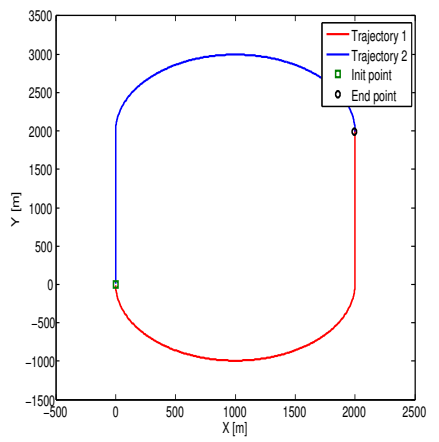

(a)

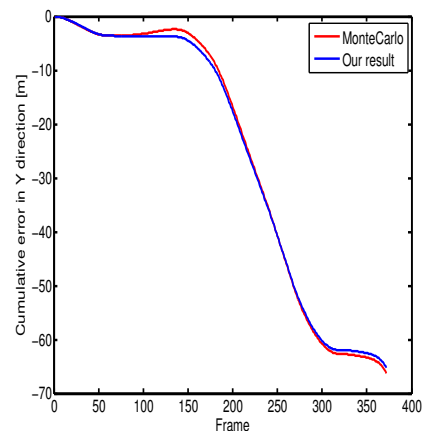

(b)

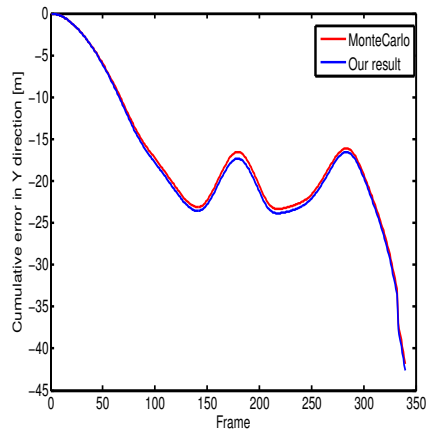

(f)

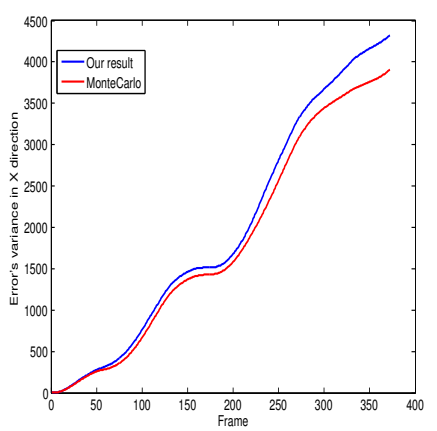

(c)

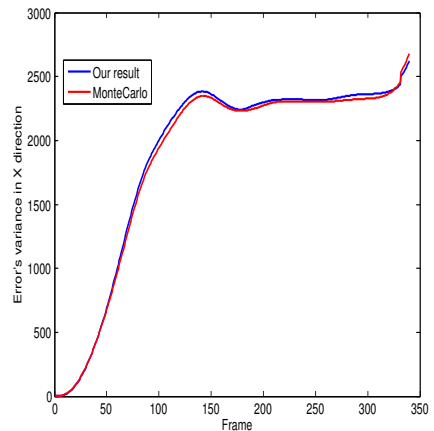

(g)

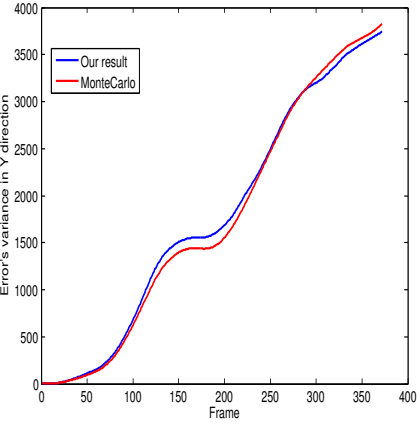

(d)

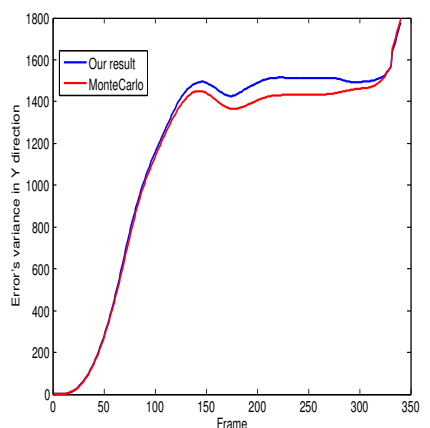

(h)

Fig. 4. Cumulative Error Estimation

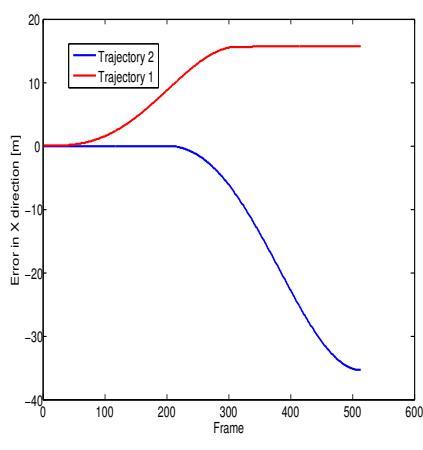

(b)

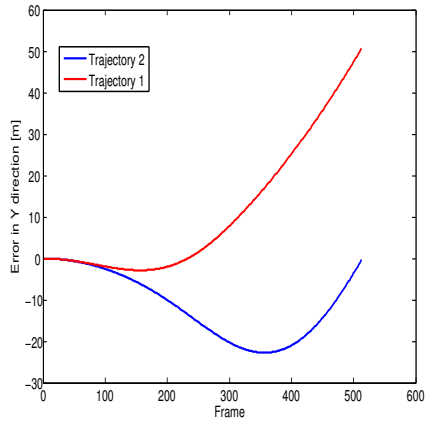

(c)

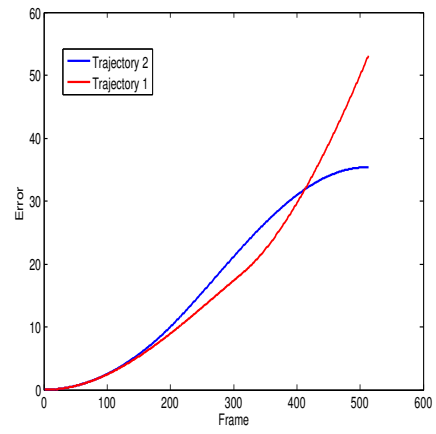

(d)

Fig. 5. Error estimation in path planning domain

\section{REFERENCES}

[1] A. Milella and R. Siegwart, "Stereo-based ego-motion estimation using pixel tracking and iterative closest point," in IEEE International Conference on Computer Vision Systems, 2006 ICVS '06, Jan. 2006, p. 21.

[2] F. Zhang, G. Chen, H. Stahle, C. Buckl, and A. Knoll, "Visual odometry based on random finite set statistics in urban environment," in Intelligent Vehicles Symposium (IV), 2012 IEEE, june 2012, pp. 69 -74 .

[3] F. Zhang, H. Stahle, A. Gaschler, C. Buckl, and A. Knoll, "Single camera visual odometry based on random finite set statistics," in Intelligent Robots and Systems (IROS), 2012 IEEE/RSJ International Conference on, 2012, pp. 559-566.

[4] F. Chenavier and J. Crowley, "Position estimation for a mobile robot using vision and odometry," in Robotics and Automation, 1992.
Proceedings., 1992 IEEE International Conference on, may 1992, pp. $2588-2593$ vol.3.

[5] T. Oskiper, Z. Zhu, S. Samarasekera, and R. Kumar, "Visual odometry system using multiple stereo cameras and inertial measurement unit," in Computer Vision and Pattern Recognition, 2007. CVPR '07. IEEE Conference on, june 2007, pp. 1 -8.

[6] J. Shen, D. Tick, and N. Gans, "Localization through fusion of discrete and continuous epipolar geometry with wheel and imu odometry," in American Control Conference (ACC), 2011, 29 2011-july 1 2011, pp. $1292-1298$.

[7] F. Zhang, S. Hauke, G. Chen, C. Chen, S. Carsten, C. Buckl, and A. Knoll, "A sensor fusion approach for localization with cumulative error elimination," in Multisensor Fusion and Integration for Intelligent Systems (MFI), 2012 IEEE Conference on, 2012, pp. 1-6.

[8] B. Stenning and T. Barfoot, "Path planning on a network of paths," in Aerospace Conference, 2011 IEEE, march 2011, pp. 1-12.

[9] I. Rekleitis, J.-L. Bedwani, S. Gemme, T. Lamarche, and E. Dupuis, 
"Terrain modelling for planetary exploration," in Computer and Robot Vision, 2007. CRV '07. Fourth Canadian Conference on, may 2007, pp. $243-249$.

[10] R. Lerner, E. Rivlin, and H. Rotstein, "Pose and motion recovery from feature correspondences and a digital terrain map," Pattern Analysis and Machine Intelligence, IEEE Transactions on, vol. 28, no. 9, pp. $1404-1417$, sept. 2006.

[11] M. Kais, S. Dauvillier, A. Foretllea, I. Masakid, and C. Laugier, "Towards outdoor localization using gis, vision system and stochastic error propagation," in International Conference on Autonomous Robots and Agents, 2004.

[12] P. Vorst and A. Zell, "Fully autonomous trajectory estimation with long-range passive rfid," in Robotics and Automation (ICRA), 2010 IEEE International Conference on, may 2010, pp. $1867-1872$.

[13] B. Kitt, A. Geiger, and H. Lategahn, "Visual odometry based on stereo image sequences with ransac-based outlier rejection scheme," in Intelligent Vehicles Symposium (IV), 2010 IEEE, june 2010, pp. 486 $-492$.

[14] R. C. Smith and P. Cheeseman, "On the representation and estimation of spatial uncertainty," The International Journal of Robotics Research, pp. 56-68, 1986.

[15] S.-F. Su and C. Lee, "Manipulation and propagation of uncertainty and verification of applicability of actions in assembly tasks," Systems, Man and Cybernetics, IEEE Transactions on, vol. 22, no. 6, pp. 1376 -1389 , nov/dec 1992.

[16] Y. Wang and G. Chirikjian, "Error propagation on the euclidean group with applications to manipulator kinematics," Robotics, IEEE Transactions on, vol. 22, no. 4, pp. 591 -602, aug. 2006.

[17] Y. Zhou and G. Chirikjian, "Probabilistic models of dead-reckoning error in nonholonomic mobile robots," in Robotics and Automation, 2003. Proceedings. ICRA '03. IEEE International Conference on, vol. 2, sept. 2003, pp. $1594-1599$ vol.2.

[18] R. Jiang, R. Klette, and S. Wang, "Modeling of unbounded long-range drift in visual odometry," in Image and Video Technology (PSIVT), 2010 Fourth Pacific-Rim Symposium on, nov. 2010, pp. 121 -126.

[19] C. Olson, L. Matthies, M. Schoppers, and M. Maimone, "Stereo ego-motion improvements for robust rover navigation," in Robotics and Automation, 2001. Proceedings 2001 ICRA. IEEE International Conference on, vol. 2, 2001, pp. 1099 - 1104 vol.2.

[20] J. Knuth and P. Barooah, "Error scaling in position estimation from noisy relative pose measurements," Mechanical and Aerospace Engineering, University of Florida, Tech. Rep., March 2011.

[21] L. Ojeda, D. Cruz, G. Reina, and J. Borenstein, "Current-based slippage detection and odometry correction for mobile robots and planetary rovers," Robotics, IEEE Transactions on, vol. 22, no. 2, pp. $366-378$, april 2006.

[22] A. Kelly, "General solution for linearized systematic error propagation in vehicle odometry," in Intelligent Robots and Systems, 2001. Proceedings. 2001 IEEE/RSJ International Conference on, vol. 4, 2001, pp. $1938-1945$ vol.4.

[23] G. Antonelli, S. Chiaverini, and G. Fusco, "An odometry calibration method for mobile robots based on the least-squares technique," in American Control Conference, 2003. Proceedings of the 2003, vol. 4, june 2003 , pp. $3429-3434$ vol.4.

[24] N. Roy and S. Thrun, "Online self-calibration for mobile robots," in Robotics and Automation, 1999. Proceedings. 1999 IEEE International Conference on, vol. 3, 1999, pp. $2292-2297$ vol.3.

\section{APPENDIX}

In order to proof the propositions, the following technical results are needed:

Assuming $\tilde{\theta} \sim N\left(0, \delta_{\theta}^{2}\right)$ and $\tilde{d} \sim N\left(0, \delta_{d}^{2}\right)$, we can obtain

$$
\begin{gathered}
\sum_{j=1}^{i} \tilde{\theta}_{j} \sim N\left(0, i \delta_{\theta}^{2}\right) ; \sum_{i=1}^{n} \tilde{d}_{i} \sim N\left(0, n \delta_{d}^{2}\right) \\
E\left(\cos \sum_{j=1}^{i} \tilde{\theta}_{j}\right)=e^{-i \frac{\delta_{\theta}^{2}}{2}}, E\left(\sin \sum_{j=1}^{i} \tilde{\theta}_{j}\right)=E\left(\sin \sum_{j=1}^{i} 2 \tilde{\theta}_{j}\right)=0 \\
E\left(\cos ^{2} \sum_{j=1}^{i} \tilde{\theta}_{j}\right)=\frac{e^{-2 i \delta_{\theta}^{2}}+1}{2}, E\left(\sin ^{2} \sum_{j=1}^{i} \tilde{\theta}_{j}\right)=\frac{-e^{-2 i \delta_{\theta}^{2}}+1}{2}
\end{gathered}
$$

Proof: Proposition 1 and 2

According to the above equations, the expectation is calculated by

$$
\begin{aligned}
& E\left[\tilde{x}_{n}\right]=\sum_{i=1}^{n} \bar{d}_{i}\left\{\sin \sum_{j=1}^{i} \bar{\theta}_{j}\left(E\left[\cos \sum_{j=1}^{i} \tilde{\theta}_{j}\right]-1\right)+\cos \sum_{j=1}^{i} \bar{\theta}_{j}\right. \\
& \left.\cdot E\left[\sin \sum_{j=1}^{i} \tilde{\theta}_{j}\right]\right\} \\
& =\sum_{i=1}^{n} \bar{d}_{i}\left[\sin \sum_{j=1}^{i} \bar{\theta}_{j}\left(e^{-\frac{i \delta_{\theta}^{2}}{2}}-1\right)\right]
\end{aligned}
$$

The variance is calculated by

$$
\operatorname{var}\left(\tilde{x}_{n}\right)=E\left[\tilde{x}^{2}\right]-E[\tilde{x}]^{2}
$$

where

$$
\begin{aligned}
& E\left[\tilde{x}^{2}\right]=E\left[\left\{\sum _ { i = 1 } ^ { n } \overline { d } _ { i } \left[\sin \sum_{j=1}^{i} \bar{\theta}_{j}\left(\cos \sum_{j=1}^{i} \tilde{\theta}_{j}-1\right)+\cos \sum_{j=1}^{i} \bar{\theta}_{j}\right.\right.\right. \\
& \left.\cdot \sin \sum_{j=1}^{i} \tilde{\theta}_{j}\right]+\sum_{i=1}^{n} \tilde{d}_{i}\left[\sin \sum_{j=1}^{i} \bar{\theta}_{j} \cos \sum_{j=1}^{i} \tilde{\theta}_{j}+\cos \sum_{j=1}^{i} \bar{\theta}_{j}\right. \\
& \left.\left.\left.\cdot \sin \sum_{j=1}^{i} \tilde{\theta}_{j}\right]\right\}^{2}\right] \\
& =E\left[\left\{\sum _ { i = 1 } ^ { n } \overline { d } _ { i } \left[\sin \sum_{j=1}^{i} \bar{\theta}_{j}\left(\cos \sum_{j=1}^{i} \tilde{\theta}_{j}-1\right)+\cos \sum_{j=1}^{i} \bar{\theta}_{j}\right.\right.\right. \\
& \left.\left.\left.\cdot \sin \sum_{j=1}^{i} \tilde{\theta}_{j}\right]\right\}^{2}\right]+E\left[\left\{\sum _ { i = 1 } ^ { n } \tilde { d } _ { i } \left[\sin \sum_{j=1}^{i} \bar{\theta}_{j} \cos \sum_{j=1}^{i} \tilde{\theta}_{j}+\cos \sum_{j=1}^{i} \bar{\theta}_{j}\right.\right.\right. \\
& \left.\left.\left.\cdot \sin \sum_{j=1}^{i} \tilde{\theta}_{j}\right]\right\}^{2}\right]+2 E\left\{\sum _ { i = 1 } ^ { n } \overline { d } _ { i } \left[\sin \sum_{j=1}^{i} \bar{\theta}_{j}\left(\cos \sum_{j=1}^{i} \tilde{\theta}_{j}-1\right)\right.\right. \\
& \left.\left.+\cos \sum_{j=1}^{i} \bar{\theta}_{j} \sin \sum_{j=1}^{i} \tilde{\theta}_{j}\right]\right\} \cdot E\left\{\sum _ { i = 1 } ^ { n } \tilde { d } _ { i } \left[\sin \sum_{j=1}^{i} \bar{\theta}_{j} \cos \sum_{j=1}^{i} \tilde{\theta}_{j}\right.\right. \\
& \left.\left.+\cos \sum_{j=1}^{i} \bar{\theta}_{j} \sin \sum_{j=1}^{i} \tilde{\theta}_{j}\right]\right\} \\
& =E\left\{\sum _ { i = 1 } ^ { n } \overline { d } _ { i } ^ { 2 } \left[\sin \sum_{j=1}^{i} \bar{\theta}_{j}\left(\cos \sum_{j=1}^{i} \tilde{\theta}_{j}-1\right)+\cos \sum_{j=1}^{i} \bar{\theta}_{j}\right.\right. \\
& \left.\left.\cdot \sin \sum_{j=1}^{i} \tilde{\theta}_{j}\right]^{2}\right\}+E\left\{2 \sum _ { i = 1 } ^ { n - 1 } \sum _ { p = 1 + i } ^ { n } \overline { d } _ { i } \left[\operatorname { s i n } \sum _ { j = 1 } ^ { i } \overline { \theta } _ { j } \left(\cos \sum_{j=1}^{i} \tilde{\theta}_{j}\right.\right.\right. \\
& \left.-1)+\cos \sum_{j=1}^{i} \bar{\theta}_{j} \sin \sum_{j=1}^{i} \tilde{\theta}_{j}\right] \cdot \bar{d}_{p}\left[\sin \sum_{k=1}^{p} \bar{\theta}_{k}\left(\cos \sum_{k=1}^{p} \tilde{\theta}_{k}-1\right)\right. \\
& \left.\left.+\cos \sum_{k=1}^{p} \bar{\theta}_{k} \sin \sum_{k=1}^{p} \tilde{\theta}_{k}\right]\right\}+E \sum_{i=1}^{n}\left\{\tilde { d } _ { i } \left[\sin \sum_{j=1}^{i} \bar{\theta}_{j} \cos \sum_{j=1}^{i} \tilde{\theta}_{j}\right.\right. \\
& \left.\left.+\cos \sum_{j=1}^{i} \bar{\theta}_{j} \sin \sum_{j=1}^{i} \tilde{\theta}_{j}\right]\right\}^{2} \\
& =E[\mathbf{A}]+E[\mathbf{B}]+E[\mathbf{C}]
\end{aligned}
$$




$$
\begin{aligned}
& E[\mathbf{A}]=E\left\{\sum _ { i = 1 } ^ { n } \overline { d } _ { i } ^ { 2 } \left[\sin \sum_{j=1}^{i} \bar{\theta}_{j}\left(\cos \sum_{j=1}^{i} \tilde{\theta}_{j}-1\right)+\cos \sum_{j=1}^{i} \bar{\theta}_{j}\right.\right. \\
& \left.\left.\cdot \sin \sum_{j=1}^{i} \tilde{\theta}_{j}\right]^{2}\right\} \\
& =\sum_{i=1}^{n} \bar{d}_{i}^{2} E\left[\sin ^{2} \sum_{j=1}^{i} \bar{\theta}_{j}\left(\cos ^{2} \sum_{j=1}^{i} \tilde{\theta}_{j}+1-2 \cos \sum_{j=1}^{i} \tilde{\theta}_{j}\right)\right. \\
& \left.+\cos ^{2} \sum_{j=1}^{i} \bar{\theta}_{j} \sin ^{2} \sum_{j=1}^{i} \tilde{\theta}_{j}\right] \\
& =\sum_{i=1}^{n} \bar{d}_{i}^{2}\left[\sin ^{2} \sum_{j=1}^{i} \bar{\theta}_{j}\left(0.5 e^{-2 i \delta_{\theta}^{2}}+1.5-2 e^{-\frac{i \delta_{\theta}^{2}}{2}}\right)+0.5\right. \text {. } \\
& \left.\cos ^{2} \sum_{j=1}^{i} \bar{\theta}_{j}\left(e^{-2 i \delta_{\theta}^{2}}+1\right)\right] \\
& E[\mathbf{B}]=E\left\{2 \sum _ { i = 1 } ^ { n - 1 } \sum _ { p = 1 + i } ^ { n } \overline { d } _ { i } \left[\sin \sum_{j=1}^{i} \bar{\theta}_{j}\left(\cos \sum_{j=1}^{i} \tilde{\theta}_{j}-1\right)+\right.\right. \\
& \left.\cos \sum_{j=1}^{i} \bar{\theta}_{j} \sin \sum_{j=1}^{i} \tilde{\theta}_{j}\right] \cdot \bar{d}_{p}\left[\sin \sum_{k=1}^{p} \bar{\theta}_{k}\left(\cos \sum_{k=1}^{p} \tilde{\theta}_{k}-1\right)+\right. \\
& \left.\left.\cos \sum_{k=1}^{p} \bar{\theta}_{k} \sin \sum_{k=1}^{p} \tilde{\theta}_{k}\right]\right\} \\
& =E\left\{2 \sum _ { i = 1 } ^ { n - 1 } \sum _ { p = 1 + i } ^ { n } \overline { d } _ { i } \left[\sin \sum_{j=1}^{i} \bar{\theta}_{j}\left(\cos \sum_{j=1}^{i} \tilde{\theta}_{j}-1\right)+\cos \sum_{j=1}^{i} \bar{\theta}_{j}\right.\right. \\
& \left.\cdot \sin \sum_{j=1}^{i} \tilde{\theta}_{j}\right] \cdot \bar{d}_{p}\left[\sin \left(\sum_{j=1}^{i} \bar{\theta}_{j}+\Delta \bar{\theta}\right)\left(\cos \left(\sum_{j=1}^{i} \tilde{\theta}_{j}+\Delta \tilde{\theta}\right)-1\right)\right. \\
& \left.-\sin \sum_{j=1}^{i} \bar{\theta}_{j} \cos \sum_{j=1}^{i} \bar{\theta}_{j} \sin \Delta \bar{\theta}_{\sin ^{2}} \sum_{j=1}^{i} \tilde{\theta}_{j} \cos \Delta \tilde{\theta}\right\} \\
& =2 \sum_{i=1}^{n} \sum_{p=1+i}^{n} \bar{d}_{i} \bar{d}_{p}\left\{\operatorname { s i n } ^ { 2 } \sum _ { j = 1 } ^ { i } \overline { \theta } _ { j } \operatorname { c o s } \Delta \overline { \theta } \left[-e^{-0.5 i \delta_{\theta}^{2}}+0.5(1+\right.\right. \\
& \left.\left.e^{-2 i \delta_{\theta}^{2}}\right) e^{-0.5 \delta_{\theta}^{2}(p-i)}+1-e^{-0.5 i \delta_{\theta}^{2}} e^{-0.5 \delta_{\theta}^{2}(p-i)}\right] \\
& +\sin \sum_{j=1}^{i} \bar{\theta}_{j} \sin \Delta \bar{\theta} \cos \sum_{j=1}^{i} \bar{\theta}_{j}\left[-e^{-0.5 i \delta_{\theta}^{2}}+0.5\left(1+e^{-2 i \delta_{\theta}^{2}}\right)\right. \\
& \cdot e^{-0.5 \delta_{\theta}^{2}(p-i)}+1-e^{-0.5 i \delta_{\theta}^{2}} e^{-0.5 \delta_{\theta}^{2}(p-i)}-0.5\left(1-e^{-2 i \delta_{\theta}^{2}}\right) \\
& \left.\cdot e^{-0.5 \delta_{\theta}^{2}(p-i)}\right]+\cos ^{2} \sum_{j=1}^{i} \bar{\theta}_{j} \cos \Delta \bar{\theta} 0.5\left(1-e^{-2 i \delta_{\theta}^{2}}\right) \\
& \left.\cdot e^{-0.5 \delta_{\theta}^{2}(p-i)}\right\} \\
& E[\mathbf{C}]=E \sum_{i=1}^{n}\left\{\tilde { d } _ { i } \left[\sin \sum_{j=1}^{i} \bar{\theta}_{j} \cos \sum_{j=1}^{i} \tilde{\theta}_{j}+\cos \sum_{j=1}^{i} \bar{\theta}_{j}\right.\right. \\
& \left.\left.\cdot \sin \sum_{j=1}^{i} \tilde{\theta}_{j}\right]\right\}^{2} \\
& =\sum_{i=1}^{n} E\left[\tilde{d}_{i}^{2}\right] E\left[\left\{\sin \sum_{j=1}^{i} \bar{\theta}_{j} \cos \sum_{j=1}^{i} \tilde{\theta}_{j}+\cos \sum_{j=1}^{i} \bar{\theta}_{j}\right.\right. \\
& \left.\left.\cdot \sin \sum_{j=1}^{i} \tilde{\theta}_{j}\right\}^{2}\right] \\
& =\sum_{i=1}^{n} E\left\{\sin ^{2} \sum_{j=1}^{i} \bar{\theta}_{j} \cos ^{2} \sum_{j=1}^{i} \tilde{\theta}_{j}+\cos ^{2} \sum_{j=1}^{i} \bar{\theta}_{j} \sin ^{2} \sum_{j=1}^{i} \tilde{\theta}_{j}\right\} \\
& =\sum_{i=1}^{n}\left[0.5 \sin ^{2} \sum_{j=1}^{i} \bar{\theta}_{j}\left(1+e^{-2 i \delta_{\theta}^{2}}\right)+0.5 \cos ^{2} \sum_{j=1}^{i} \bar{\theta}_{j}(1-\right. \\
& \left.\left.e^{-2 i \delta_{\theta}^{2}}\right)\right]
\end{aligned}
$$$$
\left.\left.+\cos \left(\sum_{j=1}^{i} \bar{\theta}_{j}+\Delta \bar{\theta}\right) \sin \left(\sum_{j=1}^{i} \tilde{\theta}_{j}+\Delta \tilde{\theta}\right)\right]\right\}
$$$$
=2 E \sum_{i=1}^{n-1} \sum_{p=1+i}^{n} \bar{d}_{i} \bar{d}_{p}\left\{\sin ^{2} \sum_{j=1}^{i} \bar{\theta}_{j} \cos ^{2} \sum_{j=1}^{i} \tilde{\theta}_{j} \cos \Delta \bar{\theta} \cos \Delta \tilde{\theta}\right.
$$$$
-\sin ^{2} \sum_{j=1}^{i} \bar{\theta}_{j} \cos \sum_{j=1}^{i} \tilde{\theta}_{j} \cos \Delta \bar{\theta}+\sin \sum_{j=1}^{i} \bar{\theta}_{j} \cos \sum_{j=1}^{i} \bar{\theta}_{j} \sin \Delta \bar{\theta}
$$$$
\cdot \cos ^{2} \sum_{j=1}^{i} \tilde{\theta}_{j} \cos \Delta \tilde{\theta}-\sin \sum_{j=1}^{i} \bar{\theta}_{j} \cos \sum_{j=1}^{i} \bar{\theta}_{j} \sin \Delta \bar{\theta} \cos \sum_{j=1}^{i} \tilde{\theta}_{j}
$$$$
-\sin ^{2} \sum_{j=1}^{i} \bar{\theta}_{j} \cos \sum_{j=1}^{i} \tilde{\theta}_{j} \cos \Delta \bar{\theta} \cos \Delta \tilde{\theta}+\sin ^{2} \sum_{j=1}^{i} \bar{\theta}_{j} \cos \Delta \bar{\theta}
$$$$
-\sin \sum_{j=1}^{i} \bar{\theta}_{j} \cos \sum_{j=1}^{i} \bar{\theta}_{j} \sin \Delta \bar{\theta} \cos \sum_{j=1}^{i} \tilde{\theta}_{j} \cos \Delta \tilde{\theta}+\sin \sum_{j=1}^{i} \bar{\theta}_{j}
$$$$
\cdot \cos \sum_{j=1}^{i} \bar{\theta}_{j} \sin \Delta \bar{\theta}+\cos ^{2} \sum_{j=1}^{i} \bar{\theta}_{j} \cos \Delta \bar{\theta}_{\sin ^{2}} \sum_{j=1}^{i} \tilde{\theta}_{j} \cos \Delta \tilde{\theta}
$$

Here $\Delta \theta$ represents the cumulative angle $\sum_{k=i+1}^{p} \theta_{k}$. The error's expectation and variance in $y$ direction can also be estimated by the same process.

With the same manner, proposition 2 can also be proofed by arranging the following equations:

$$
\bar{d}=d^{m}-\tilde{d}, \bar{\theta}=\theta^{m}-\tilde{\theta}
$$

Due to the page limit, the proof process is omitted here. 